\title{
CONVERGENCE OF MULTISPLITTING METHODS WITH PREWEIGHTING FOR AN $H$-MATRIX
}

\author{
Yu Du Han and Jae HeOn Yun
}

\begin{abstract}
In this paper, we study convergence of multisplitting methods with preweighting for solving a linear system whose coefficient matrix is an $H$-matrix corresponding to both the AOR multisplitting and the SSOR multisplitting. Numerical results are also provided to confirm theoretical results for the convergence of multisplitting methods with preweighting.
\end{abstract}

\section{Introduction}

In this paper, we consider multisplitting methods with preweighting for solving a linear system of the form

$$
A x=b, \quad x, b \in \mathbb{R}^{n},
$$

where $A \in \mathbb{R}^{n \times n}$ is a large sparse nonsingular matrix. Multisplitting method was first introduced by O'Leary and White [6] for parallel computation of the linear system (1).

For a vector $x \in \mathbb{R}^{n}, x \geq 0(x>0)$ denotes that all components of $x$ are nonnegative (positive), and $|x|$ denotes the vector whose components are the absolute values of the corresponding components of $x$. For two vectors $x, y \in \mathbb{R}^{n}, x \geq y(x>y)$ means that $x-y \geq 0(x-y>0)$. These definitions carry immediately over to matrices. For a square matrix $A, \operatorname{diag}(A)$ denotes a diagonal matrix whose diagonal part coincides with the diagonal part of $A$. Let $\rho(A)$ denote the spectral radius of a square matrix $A$. Varga [8] showed that for any two square matrices $A$ and $B,|A| \leq B$ implies $\rho(A) \leq \rho(B)$.

A matrix $A=\left(a_{i j}\right) \in \mathbb{R}^{n \times n}$ is called monotone if $A$ is nonsingular with $A^{-1} \geq 0$. A matrix $A=\left(a_{i j}\right) \in \mathbb{R}^{n \times n}$ is called an $M$-matrix if it is a monotone matrix with $a_{i j} \leq 0$ for $i \neq j$. The comparison matrix $\langle A\rangle=\left(\alpha_{i j}\right)$ of a matrix $A=\left(a_{i j}\right)$ is defined by

$$
\alpha_{i j}=\left\{\begin{aligned}
\left|a_{i j}\right| & \text { if } i=j, \\
-\left|a_{i j}\right| & \text { if } i \neq j .
\end{aligned}\right.
$$

Received May 19, 2011; Revised November 15, 2011.

2010 Mathematics Subject Classification. 65F10, 65F15.

Key words and phrases. multisplitting method, preweighting, AOR multisplitting, SSOR multisplitting, $H$-matrix. 
A matrix $A$ is called an $H$-matrix if $\langle A\rangle$ is an $M$-matrix.

A representation $A=M-N$ is called a splitting of $A$ if $M$ is nonsingular. A splitting $A=M-N$ is called regular if $M^{-1} \geq 0$ and $N \geq 0$, and called weak regular if $M^{-1} \geq 0$ and $M^{-1} N \geq 0$ [1]. It is well known that if $A=M-N$ is a weak regular splitting of $\mathrm{A}$, then $\rho\left(M^{-1} N\right)<1$ if and only if $A^{-1} \geq 0[1,8]$. A collection of triples $\left(M_{k}, N_{k}, E_{k}\right), k=1,2, \ldots, \ell$, is called a multisplitting of $A$ if $A=M_{k}-N_{k}$ is a splitting of $A$ for $k=1,2, \ldots, \ell$, and $E_{k}$ 's, called weighting matrices, are nonnegative diagonal matrices such that $\sum_{k=1}^{\ell} E_{k}=I$.

The multisplitting method with postweighting which is usually called the multisplitting method has been extensively studied in the literature, see $[2,3,4$, $5,6,7,9,11,12]$. In 1989, White [10] proposed multisplitting method with different weighting schemes, and he showed that multisplitting method with preweighting yields the fastest method in certain situations. However, the multisplitting method with preweighting has not been studied extensively, see $[4,10]$. This is the main motivation for studying convergence of multisplitting method with preweighting. The purpose of this paper is to study convergence of multisplitting methods with preweighting for solving a linear system whose coefficient matrix is an $H$-matrix corresponding to both the AOR multisplitting and the SSOR multisplitting. We also provide numerical results to confirm theoretical results for the convergence of multisplitting methods with preweighting.

\section{Convergence of multisplitting methods with preweighting}

Let $\left(M_{k}, N_{k}, E_{k}\right), k=1,2, \ldots, \ell$, be a multisplitting of $\mathrm{A}$. Then the corresponding multisplitting method with preweighting for solving $A x=b[10]$ is given by

$$
\begin{aligned}
x_{i+1} & =H_{0} x_{i}+G_{0} b \\
& =x_{i}+G_{0}\left(b-A x_{i}\right), i=0,1,2, \ldots,
\end{aligned}
$$

where

$$
G_{0}=\sum_{k=1}^{\ell} M_{k}^{-1} E_{k} \text { and } H_{0}=I-G_{0} A .
$$

$H_{0}=I-\sum_{k=1}^{\ell} M_{k}{ }^{-1} E_{k} A$ is called an iteration matrix for the multisplitting method with preweighting. Notice that $H=I-\sum_{k=1}^{\ell} E_{k} M_{k}{ }^{-1} A$ is called an iteration matrix for the multisplitting method. By simple calculation, one obtains

$$
H_{0}^{T}=A^{T}\left(I-\sum_{k=1}^{\ell} E_{k}\left(M_{k}^{T}\right)^{-1} A^{T}\right)\left(A^{T}\right)^{-1} .
$$

Let $\hat{H}=I-\sum_{k=1}^{\ell} E_{k}\left(M_{k}^{T}\right)^{-1} A^{T}=\sum_{k=1}^{\ell} E_{k}\left(M_{k}^{T}\right)^{-1} N_{k}^{T}$. Then $\hat{H}$ is similar to $H_{0}{ }^{T}$ and hence $\rho\left(H_{0}\right)=\rho(\hat{H})$. Notice that $\hat{H}$ is an iteration matrix for the multisplitting method corresponding to a multisplitting $\left(M_{k}^{T}, N_{k}^{T}, E_{k}\right)$, 
$k=1,2, \ldots, \ell$, of $A^{T}$. Hence, convergence result of multisplitting method with preweighting corresponding to a multisplitting of $A$ can be obtained from that of multisplitting method corresponding to a multisplitting of $A^{T}$.

The multisplitting method with preweighting associated with a multisplitting $\left(M_{k}, N_{k}, E_{k}\right), k=1,2, \ldots, \ell$, of $A$ for solving the linear system (1) is as follows:

Algorithm 1: Multisplitting Method With PReweighting

Given an initial vector $x_{0}$

For $i=0,1, \ldots$, until convergence

For $k=1$ to $\ell$ \{parallel execution\}

$M_{k} y_{k}=E_{k}\left(b-A x_{i}\right)$

$x_{i+1}=x_{i}+\sum_{k=1}^{\ell} y_{k}$

From now on, it is assumed that $A=D-L_{k}-V_{k}$, where $D=\operatorname{diag}(A)$ is a nonsingular matrix, $L_{k}$ is a strictly lower triangular matrix and $V_{k}$ is a general matrix for $k=1,2, \ldots, \ell$. The AOR-multisplitting method with preweighting is defined by

$$
x_{i+1}=H_{0}(\omega, \gamma) x_{i}+G_{0}(\omega, \gamma) b, i=0,1,2, \ldots,
$$

where

$$
\begin{aligned}
& G_{0}(\omega, \gamma)=\omega \sum_{k=1}^{\ell}\left(D-\gamma L_{k}\right)^{-1} E_{k}, \\
& H_{0}(\omega, \gamma)=I-\omega \sum_{k=1}^{\ell}\left(D-\gamma L_{k}\right)^{-1} E_{k} A .
\end{aligned}
$$

Notice that $\omega A=\left(D-\gamma L_{k}\right)-\left((1-\omega) D+(\omega-\gamma) L_{k}+\omega V_{k}\right)$ for $k=1,2, \ldots, \ell$ and

$$
H_{0}(\omega, \gamma)^{T}=A^{T}\left(I-\omega \sum_{k=1}^{\ell} E_{k}\left(D-\gamma L_{k}^{T}\right)^{-1} A^{T}\right) A^{-T} .
$$

Let $\tilde{H}(\omega, \gamma)=I-\omega \sum_{k=1}^{\ell} E_{k}\left(D-\gamma L_{k}{ }^{T}\right)^{-1} A^{T}$. Then $\tilde{H}(\omega, \gamma)$ is similar to $H_{0}(\omega, \gamma)^{T}$ and $\tilde{H}(\omega, \gamma)$ can be written as

$$
\tilde{H}(\omega, \gamma)=\sum_{k=1}^{\ell} E_{k}\left(D-\gamma L_{k}^{T}\right)^{-1}\left((1-\omega) D+(\omega-\gamma) L_{k}^{T}+\omega V_{k}^{T}\right) .
$$

Notice that $\tilde{H}(\omega, \gamma)$ is an iteration matrix of the multisplitting method corresponding to a multisplitting

$$
\left(\frac{1}{\omega}\left(D-\gamma L_{k}^{T}\right), \frac{1}{\omega}\left((1-\omega) D+(\omega-\gamma) L_{k}^{T}+\omega V_{k}^{T}\right), E_{k}\right), k=1,2, \ldots, \ell
$$


of $A^{T}$. The following lemma provides a convergence result of the multisplitting method corresponding to a multisplitting

$$
\left(\frac{1}{\omega}\left(D-\gamma L_{k}^{T}\right), \frac{1}{\omega}\left((1-\omega) D+(\omega-\gamma) L_{k}^{T}+\omega V_{k}^{T}\right), E_{k}\right), k=1,2, \ldots, \ell
$$

of $A^{T}$ when $A$ is an $H$-matrix.

Lemma 2.1. Let $A=D-B$ be an $n \times n H$-matrix with $D=\operatorname{diag}(A)$. Let $A=D-L_{k}-V_{k}$, where $L_{k}$ is a strictly lower triangular matrix and $V_{k}$ is a general matrix for $k=1,2, \ldots, \ell$. Assume that $\langle A\rangle=|D|-\left|L_{k}\right|-\left|V_{k}\right|$ for $k=$ $1,2, \ldots, \ell$. If $0<\gamma \leq \omega<\frac{2}{1+\alpha}$, then $\rho(\tilde{H}(\omega, \gamma))<1$, where $\alpha=\rho\left(|D|^{-1}|B|\right)$ and

$$
\tilde{H}(\omega, \gamma)=\sum_{k=1}^{\ell} E_{k}\left(D-\gamma L_{k}^{T}\right)^{-1}\left((1-\omega) D+(\omega-\gamma) L_{k}^{T}+\omega V_{k}^{T}\right) .
$$

Proof. From the assumption, $\left\langle A^{T}\right\rangle=|D|-\left|L_{k}^{T}\right|-\left|V_{k}^{T}\right|$ for $k=1,2, \ldots, \ell$. Notice that for $k=1,2, \ldots, \ell$,

(6) $\quad \omega\left\langle A^{T}\right\rangle=\left(|D|-\gamma\left|L_{k}^{T}\right|\right)-\left((1-\omega)|D|+(\omega-\gamma)\left|L_{k}^{T}\right|+\omega\left|V_{k}^{T}\right|\right)$.

Since $D-\gamma L_{k}$ is an $H$-matrix, $\left(D-\gamma L_{k}\right)^{T}$ is also an $H$-matrix. Consider the following inequality

$$
\begin{aligned}
|\tilde{H}(\omega, \gamma)| & \leq \sum_{k=1}^{\ell} E_{k}\left\langle D-\gamma L_{k}^{T}\right\rangle^{-1}\left(|1-\omega||D|+(\omega-\gamma)\left|L_{k}^{T}\right|+\omega\left|V_{k}^{T}\right|\right) \\
& =\sum_{k=1}^{\ell} E_{k}\left(|D|-\gamma\left|L_{k}^{T}\right|\right)^{-1}\left(|1-\omega||D|+(\omega-\gamma)\left|L_{k}^{T}\right|+\omega\left|V_{k}^{T}\right|\right)
\end{aligned}
$$

Let we define

$$
H^{\star}(\omega, \gamma)=\sum_{k=1}^{\ell} E_{k}\left(|D|-\gamma\left|L_{k}^{T}\right|\right)^{-1}\left(|1-\omega||D|+(\omega-\gamma)\left|L_{k}^{T}\right|+\omega\left|V_{k}^{T}\right|\right) .
$$

Then we have

$$
|\tilde{H}(\omega, \gamma)| \leq H^{\star}(\omega, \gamma)
$$

We first consider the case where $0<\gamma \leq \omega \leq 1$. Since $\left(|D|-\gamma\left|L_{k}^{T}\right|\right)^{-1} \geq 0$ and $(1-\omega)|D|+(\omega-\gamma)\left|L_{k}^{T}\right|+\omega\left|V_{k}^{T}\right| \geq 0,(6)$ is a regular splitting of $\omega\left\langle A^{T}\right\rangle$ for each $k=1,2, \ldots, \ell$. Since $\left\langle A^{T}\right\rangle^{-1} \geq 0, \rho\left(H^{\star}(\omega, \gamma)\right)<1$. From $(7), \rho(\tilde{H}(\omega, \gamma))<1$ for $0<\gamma \leq \omega \leq 1$. Next we consider the case where $1<\omega<\frac{2}{1+\alpha}$ and $\gamma \leq \omega$. Let

$$
\begin{aligned}
\tilde{N}_{k}(\omega, \gamma) & =(\omega-1)|D|+(\omega-\gamma)\left|L_{k}^{T}\right|+\omega\left|V_{k}^{T}\right|(1 \leq k \leq \ell), \\
\tilde{A} & =(2-\omega)|D|-\omega\left|B^{T}\right| .
\end{aligned}
$$


It is easy to show that $\tilde{A}=\left(|D|-\gamma\left|L_{k}{ }^{T}\right|\right)-\tilde{N}_{k}(\omega, \gamma)$ for all $k=1,2, \ldots, \ell$. By simple calculation, one obtains

$$
|\tilde{H}(\omega, \gamma)| \leq \sum_{k=1}^{\ell} E_{k}\left(|D|-\gamma\left|L_{k}^{T}\right|\right)^{-1} \tilde{N}_{k}(\omega, \gamma)
$$

Since $\omega<\frac{2}{1+\alpha}, \frac{\omega \alpha}{2-\omega}<1$ and thus

$$
\frac{\omega}{2-\omega} \rho\left(|D|^{-1}\left|B^{T}\right|\right)=\frac{\omega}{2-\omega} \rho\left(|D|^{-1}|B|\right)=\frac{\omega \alpha}{2-\omega}<1 .
$$

Since $\tilde{A}=(2-\omega)|D|-\omega\left|B^{T}\right|$ is a regular splitting of $\tilde{A}, \tilde{A}^{-1} \geq 0$. Since $\tilde{A}=\left(|D|-\gamma\left|L_{k}^{T}\right|\right)-\tilde{N}_{k}(\omega, \gamma)$ is a regular splitting of $\tilde{A}$ for each $k=1,2, \ldots, \ell$, $\rho\left(\sum_{k=1}^{\ell} E_{k}\left(|D|-\gamma\left|L_{k}^{T}\right|\right)^{-1} \tilde{N}_{k}(\omega, \gamma)\right)<1$. From $(8), \rho(\tilde{H}(\omega, \gamma))<1$ for $1<\omega<\frac{2}{1+\alpha}$ and $\gamma \leq \omega$. Therefore, $\rho(\tilde{H}(\omega, \gamma))<1$ for $0<\gamma \leq \omega<\frac{2}{1+\alpha}$.

The following theorem provides a convergence result of the AOR-multisplitting method with preweighting when $A$ is an $H$-matrix.

Theorem 2.2. Let $A=D-B$ be an $n \times n H$-matrix with $D=\operatorname{diag}(A)$. Let $A=D-L_{k}-V_{k}$, where $L_{k}$ is a strictly lower triangular matrix and $V_{k}$ is a general matrix for $k=1,2, \ldots, \ell$. Assume that $\langle A\rangle=|D|-\left|L_{k}\right|-\left|V_{k}\right|$ for $k=1,2, \ldots, \ell$. If $0<\gamma \leq \omega<\frac{2}{1+\alpha}$, then

$$
\rho\left(H_{0}(\omega, \gamma)\right)<1
$$

where $H_{0}(\omega, \gamma)=I-\omega \sum_{k=1}^{\ell}\left(D-\gamma L_{k}\right)^{-1} E_{k} A$ and $\alpha=\rho\left(|D|^{-1}|B|\right)$.

Proof. Let $\tilde{H}(\omega, \gamma)=I-\omega \sum_{k=1}^{\ell} E_{k}\left(D-\gamma L_{k}^{T}\right)^{-1} A^{T}$. Since $\tilde{H}(\omega, \gamma)$ is similar to $H_{0}(\omega, \gamma)^{T}, \rho(\tilde{H}(\omega, \gamma))=\rho\left(H_{0}(\omega, \gamma)\right)$. From Lemma 2.1, $\rho(\tilde{H}(\omega, \gamma))<1$ for $0<\gamma \leq \omega<\frac{2}{1+\alpha}$. Therefore, $\rho\left(H_{0}(\omega, \gamma)\right)<1$ for $0<\gamma \leq \omega<\frac{2}{1+\alpha}$.

Notice that if $A$ is an $M$-matrix, then $A$ is an $H$-matrix. We easily obtain the following corollary which is a convergence results of the AOR-multisplitting method with preweighting when $A$ is an $M$-matrix.

Corollary 2.3. Let $A=D-B$ be an $n \times n M$-matrix with $D=\operatorname{diag}(A)$. Let $A=D-L_{k}-V_{k}$, where $L_{k} \geq 0$ is a strictly lower triangular matrix and $V_{k} \geq 0$ is a general matrix for $k=1,2, \ldots, \ell$. If $0<\gamma \leq \omega<\frac{2}{1+\alpha}$, then

$$
\rho\left(H_{0}(\omega, \gamma)\right)<1
$$

where $H_{0}(\omega, \gamma)=I-\omega \sum_{k=1}^{\ell}\left(D-\gamma L_{k}\right)^{-1} E_{k} A$ and $\alpha=\rho\left(D^{-1} B\right)$.

The SSOR-multisplitting method with preweighting is defined by

$$
x_{i+1}=H_{0}(\omega) x_{i}+G_{0}(\omega) b, i=0,1,2, \ldots,
$$


where

$$
\begin{aligned}
& G_{0}(\omega)=\omega(2-\omega) \sum_{k=1}^{\ell}\left(\left(D-\omega L_{k}\right) D^{-1}\left(D-\omega V_{k}\right)\right)^{-1} E_{k}, \\
& H_{0}(\omega)=I-\omega(2-\omega) \sum_{k=1}^{\ell}\left(\left(D-\omega L_{k}\right) D^{-1}\left(D-\omega V_{k}\right)\right)^{-1} E_{k} A .
\end{aligned}
$$

Notice that for $k=1,2, \ldots, \ell$,

$$
\begin{aligned}
\omega(2-\omega) A= & \left(D-\omega L_{k}\right) D^{-1}\left(D-\omega V_{k}\right) \\
& -\left((1-\omega) D+\omega L_{k}\right) D^{-1}\left((1-\omega) D+\omega V_{k}\right)
\end{aligned}
$$

and

$$
H_{0}(\omega)^{T}=A^{T}\left(I-\omega(2-\omega) \sum_{k=1}^{\ell} E_{k}\left(\left(D-\omega L_{k}\right) D^{-1}\left(D-\omega V_{k}\right)\right)^{-T} A^{T}\right) A^{-T} .
$$

Let $\tilde{H}(\omega)=I-\omega(2-\omega) \sum_{k=1}^{\ell} E_{k}\left(\left(D-\omega L_{k}\right) D^{-1}\left(D-\omega V_{k}\right)\right)^{-T} A^{T}$. Then $\tilde{H}(\omega)$ is similar to $H_{0}(\omega)^{T}$ and $\tilde{H}(\omega)$ can be written as

$$
\widetilde{H}(\omega)=\sum_{k=1}^{\ell} E_{k} M_{k}(\omega)^{-T} N_{k}(\omega)^{T}
$$

where $M_{k}(\omega)=\left(D-\omega L_{k}\right) D^{-1}\left(D-\omega V_{k}\right)$ and $N_{k}(\omega)=\left((1-\omega) D+\omega L_{k}\right) D^{-1}((1$ $\left.-\omega) D+\omega V_{k}\right)$. Note that $\tilde{H}(\omega)$ is an iteration matrix of multisplitting method corresponding to a multisplitting

$$
\left(\frac{1}{\omega(2-\omega)} M_{k}(\omega)^{T}, \frac{1}{\omega(2-\omega)} N_{k}(\omega)^{T}, E_{k}\right), k=1,2, \ldots, \ell
$$

of $A^{T}$. The following theorem provides a convergence result of the SSORmultisplitting method with preweighting when $A$ is an $H$-matrix.

Theorem 2.4. Let $A=D-B$ be an $n \times n H$-matrix with $D=\operatorname{diag}(A)$. Let $A=D-L_{k}-V_{k}$, where $L_{k}$ is a strictly lower triangular matrix and $V_{k}$ is a general matrix for $k=1,2, \ldots, \ell$. Assume that $\langle A\rangle=|D|-\left|L_{k}\right|-\left|V_{k}\right|$ for $k=1,2, \ldots, \ell$. If $0<\omega<\frac{2}{1+\alpha}$, then

$$
\rho\left(H_{0}(\omega)\right)<1,
$$

where $H_{0}(\omega)=I-\omega(2-\omega) \sum_{k=1}^{\ell}\left(\left(D-\omega L_{k}\right) D^{-1}\left(D-\omega V_{k}\right)\right)^{-1} E_{k} A$ and $\alpha=$ $\rho\left(|D|^{-1}|B|\right)$.

Proof. Let $\tilde{H}(\omega)=I-\omega(2-\omega) \sum_{k=1}^{\ell} E_{k}\left(\left(D-\omega L_{k}\right) D^{-1}\left(D-\omega V_{k}\right)\right)^{-T} A^{T}$. Then, $\tilde{H}(\omega)$ is similar to $H_{0}(\omega)^{T}$ and thus $\rho(\tilde{H}(\omega))=\rho\left(H_{0}(\omega)\right)$. Hence, it is 
sufficient to show that $\rho(\tilde{H}(\omega))<1$. Notice that $\left(D-\omega L_{k}\right)^{T}$ and $\left(D-\omega V_{k}\right)^{T}$ are $H$-matrices since $0<\omega<\frac{2}{1+\alpha}$ and $\alpha<1$. For $k=1,2, \ldots, \ell$, let

$$
\begin{aligned}
\tilde{M}_{k}(\omega) & =\left(|D|-\omega\left|U_{k}^{T}\right|\right)\left|D^{-1}\right|\left(|D|-\omega\left|L_{k}^{T}\right|\right), \\
\tilde{N}_{k}(\omega) & =\left(|1-\omega||D|+\omega\left|U_{k}^{T}\right|\right)\left|D^{-1}\right|\left(|1-\omega||D|+\omega\left|L_{k}^{T}\right|\right), \\
H^{*}(\omega) & =\sum_{k=1}^{\ell} E_{k} \tilde{M}_{k}(\omega)^{-1} \tilde{N}_{k}(\omega), \\
\tilde{A}(\omega) & =\tilde{M}_{k}(\omega)-\tilde{N}_{k}(\omega) .
\end{aligned}
$$

Then it can be easily shown that

$$
|\tilde{H}(\omega)| \leq H^{*}(\omega) .
$$

We first consider the case where $0<\omega \leq 1$. By simple calculation, $\tilde{A}(\omega)=$ $\omega(2-\omega)\langle A\rangle^{T}$. Since $\tilde{A}(\omega)=\tilde{M}_{k}(\omega)-\tilde{N}_{k}(\omega)$ is a regular splitting of $\tilde{A}(\omega)$ and $\tilde{A}(\omega)^{-1} \geq 0, \rho\left(H^{*}(\omega)\right)<1$. From (11), $\rho(\tilde{H}(\omega))<1$ for $0<\omega \leq 1$. We next consider the case where $1<\omega<\frac{2}{1+\alpha}$. By simple calculation, $\tilde{A}(\omega)=$ $\omega(2-\omega)|D|-\omega^{2}\left|B^{T}\right|$. Since $\omega<\frac{2}{1+\alpha}, \frac{\omega \alpha}{2-\omega}<1$ and thus

$$
\frac{\omega^{2}}{\omega(2-\omega)} \rho\left(|D|^{-1}\left|B^{T}\right|\right)=\frac{\omega}{2-\omega} \rho\left(|D|^{-1}|B|\right)<1 .
$$

Hence $\tilde{A}(\omega)^{-1} \geq 0$. Since $\tilde{A}(\omega)=\tilde{M}_{k}(\omega)-\tilde{N}_{k}(\omega)$ is a regular splitting of $\tilde{A}(\omega), \rho\left(H^{*}(\omega)\right)<1$. From (11), $\rho(\tilde{H}(\omega))<1$ for $1<\omega<\frac{2}{1+\alpha}$. Therefore, $\rho(\tilde{H}(\omega))<1$ for $0<\omega<\frac{2}{1+\alpha}$.

Corollary 2.5. Let $A=D-B$ be an $n \times n M$-matrix with $D=\operatorname{diag}(A)$. Let $A=D-L_{k}-V_{k}$, where $L_{k} \geq 0$ is a strictly lower triangular matrix and $V_{k} \geq 0$ is a general matrix for $k=1,2, \ldots$, $\ell$. If $0<\omega<\frac{2}{1+\alpha}$, then

$$
\rho\left(H_{0}(\omega)\right)<1,
$$

where $H_{0}(\omega)=I-\omega(2-\omega) \sum_{k=1}^{\ell}\left(\left(D-\omega L_{k}\right) D^{-1}\left(D-\omega V_{k}\right)\right)^{-1} E_{k} A$ and $\alpha=$ $\rho\left(D^{-1} B\right)$.

We now provide numerical results to illustrate the convergence of the multisplitting methods with preweighting. All numerical values are computed using MATLAB.

Example 2.6. Suppose that $\ell=3$. Consider an $H$-matrix $A$ of the form

$$
A=\left(\begin{array}{rrr}
F & I & 0 \\
-I & F & I \\
0 & -I & F
\end{array}\right) \text {, where } F=\left(\begin{array}{rrr}
4 & -1 & 0 \\
1 & 4 & -1 \\
0 & 1 & 4
\end{array}\right) \text { and } I=\left(\begin{array}{ccc}
1 & 0 & 0 \\
0 & 1 & 0 \\
0 & 0 & 1
\end{array}\right) \text {. }
$$


TABLE 1. Numerical values of $\rho\left(H_{0}(\omega, \gamma)\right)$ for Example 2.6

\begin{tabular}{|ccc|ccc|}
\hline$\omega$ & $\gamma$ & $\rho\left(H_{0}(\omega, \gamma)\right)$ & $\omega$ & $\gamma$ & $\rho\left(H_{0}(\omega, \gamma)\right)$ \\
\hline 1.17 & 1.17 & 0.8470 & 0.9 & 0.9 & $\mathbf{0 . 2 6 4 7}$ \\
& 1.0 & 0.6542 & & 0.8 & 0.2880 \\
& 0.8 & $\mathbf{0 . 5 7 1 6}$ & & 0.7 & 0.3384 \\
& 0.7 & 0.5889 & 0.8 & 0.8 & $\mathbf{0 . 2 5 4 0}$ \\
& 0.6 & 0.6215 & & 0.7 & 0.2946 \\
1.1 & 1.1 & 0.6214 & & 0.6 & 0.3517 \\
& 1.0 & 0.5574 & 0.7 & 0.7 & $\mathbf{0 . 3 3 0 6}$ \\
& 0.9 & 0.5043 & & 0.6 & 0.3539 \\
& 0.8 & $\mathbf{0 . 4 9 0 4}$ & & 0.5 & 0.4002 \\
& 0.7 & 0.5143 & 0.6 & 0.6 & $\mathbf{0 . 4 1 5 9}$ \\
& 0.6 & 0.5516 & & 0.5 & 0.4344 \\
1.0 & 1.0 & 0.4204 & & 0.4 & 0.4683 \\
& 0.9 & $\mathbf{0 . 3 8 0 0}$ & 0.5 & 0.5 & $\mathbf{0 . 5 0 7 6}$ \\
& 0.8 & 0.3812 & & 0.4 & 0.5232 \\
& 0.7 & 0.4168 & & 0.3 & 0.5472 \\
\hline
\end{tabular}

TABLE 2. Numerical values of $\rho\left(H_{0}(\omega)\right)$ for Example 2.6

\begin{tabular}{|cc|cc|}
\hline$\omega$ & $\rho\left(H_{0}(\omega)\right)$ & $\omega$ & $\rho\left(H_{0}(\omega)\right)$ \\
\hline 1.17 & 0.1603 & 0.7 & 0.1353 \\
1.1 & 0.1279 & 0.6 & 0.1939 \\
1.0 & 0.1014 & 0.5 & 0.2728 \\
0.9 & 0.1172 & 0.4 & 0.3731 \\
0.8 & $\mathbf{0 . 1 0 0 0}$ & 0.3 & 0.4961 \\
\hline
\end{tabular}

Let $F=D_{*}-L_{*}-U_{*}$, where $D_{*}=\operatorname{diag}(F)$,

$$
L_{*}=\left(\begin{array}{rrr}
0 & 0 & 0 \\
-1 & 0 & 0 \\
0 & -1 & 0
\end{array}\right) \text {, and } U_{*}=\left(\begin{array}{lll}
0 & 1 & 0 \\
0 & 0 & 1 \\
0 & 0 & 0
\end{array}\right) \text {. }
$$

Let $D=\operatorname{diag}(A), B=D-A$,

$$
\begin{array}{rlrl}
L_{1}= & \left(\begin{array}{ccc}
L_{*} & 0 & 0 \\
I & L_{*} & 0 \\
0 & 0 & L_{*}
\end{array}\right), & L_{2}=\left(\begin{array}{ccc}
L_{*} & 0 & 0 \\
0 & L_{*} & 0 \\
0 & I & L_{*}
\end{array}\right), L_{3}=\left(\begin{array}{ccc}
L_{*} & 0 & 0 \\
0 & L_{*} & 0 \\
0 & 0 & L_{*}
\end{array}\right), \\
V_{1}=\left(\begin{array}{ccc}
U_{*} & -I & 0 \\
0 & U_{*} & -I \\
0 & I & U_{*}
\end{array}\right), V_{2}=\left(\begin{array}{ccc}
U_{*} & -I & 0 \\
I & U_{*} & -I \\
0 & 0 & U_{*}
\end{array}\right), \quad V_{3}=\left(\begin{array}{ccc}
U_{*} & -I & 0 \\
I & U_{*} & -I \\
0 & I & U_{*}
\end{array}\right), \\
E_{1}=\left(\begin{array}{lll}
I & 0 & 0 \\
0 & 0 & 0 \\
0 & 0 & 0
\end{array}\right), E_{2}=\left(\begin{array}{ccc}
0 & 0 & 0 \\
0 & I & 0 \\
0 & 0 & 0
\end{array}\right), \text { and } E_{3}=\left(\begin{array}{ccc}
0 & 0 & 0 \\
0 & 0 & 0 \\
0 & 0 & I
\end{array}\right) .
\end{array}
$$


Then, $A=D-L_{k}-U_{k}$ and $\langle A\rangle=|D|-\left|L_{k}\right|-\left|U_{k}\right|$ for $k=1,2,3$. The iteration matrix for the AOR-multisplitting method with preweighting is $H_{0}(\omega, \gamma)=I-$ $\omega \sum_{k=1}^{\ell}\left(D-\gamma L_{k}\right)^{-1} E_{k} A$, and the iteration matrix for the SSOR-multisplitting method with preweighting is given by

$$
H_{0}(\omega)=I-\omega(2-\omega) \sum_{k=1}^{\ell}\left(\left(D-\omega L_{k}\right) D^{-1}\left(D-\omega V_{k}\right)\right)^{-1} E_{k} A .
$$

Note that $\alpha=\rho\left(|D|^{-1}|B|\right) \approx 0.7071$ and $\frac{2}{1+\alpha} \approx 1.1716$. For various values of $\omega$ and $\gamma$, the numerical values of $\rho\left(H_{0}(\omega, \gamma)\right)$ are listed in Table 1 . For various values of $\omega$, the numerical values of $\rho\left(H_{0}(\omega)\right)$ are listed in Table 2.

From Tables 1 and 2, it can be seen that numerical results are in good agreement with the theoretical results obtained in this section. For Example 2.6, the AOR-multisplitting method with preweighting performs best for about $\omega=\gamma=0.8$, and the SSOR-multisplitting method with preweighting performs best for about $\omega=0.8$. Notice that the optimal values of $\omega$ and $\gamma$ vary depending upon the problem. Future work will include theoretical study for finding optimal values of $\omega$ and $\gamma$ for which these multisplitting methods perform best.

Acknowledgements. The authors would like to thank anonymous referees for their useful suggestions which improved the quality of this paper.

\section{References}

[1] A. Berman and R. J. Plemmons, Nonnegative Matrices in the Mathematical Sciences, Academic Press, New York, 1979.

[2] Z. H. Cao and Z. Y. Liu, Convergence of relaxed parallel multisplitting methods with different weighting schemes, Appl. Math. Comp. 106 (1999), no. 2-3, 181-196.

[3] A. Frommer and G. Mayer, Convergence of relaxed parallel multisplitting methods, Linear Algebra Appl. 119 (1989), 141-152.

[4] - On the theory and practice of multisplitting methods in parallel computation, Computing 49 (1992), no. 1, 63-74.

[5] M. Neumann and R. J. Plemmons, Convergence of parallel multisplitting iterative methods for $M$-matrices, Linear Algebra Appl. 88/89 (1987), 559-573.

[6] D. P. O'Leary and R. E. White, Multisplittings of matrices and parallel solution of linear systems, SIAM J. Algebraic Discrete Methods 6 (1985), no. 4, 630-640.

[7] D. B. Szyld and M. T. Jones, Two-stage and multisplitting methods for the parallel solution of linear systems, SIAM J. Matrix Anal. Appl. 13 (1992), no. 2, 671-679.

[8] R. S. Varga, Matrix Iterative Analysis, Springer, Berlin, 2000.

[9] C. L. Wang and J. H. Zhao, Further results on regular splittings and multisplittings, Int. J. Comput. Math. 82 (2005), no. 4, 421-431.

[10] R. E. White, Multisplitting with different weighting schemes, SIAM J. Matrix Anal. Appl. 10 (1989), no. 4, 481-493.

[11] _ Multisplitting of a symmetric positive definite matrix, SIAM J. Matrix Anal. Appl. 11 (1990), no. 1, 69-82.

[12] J. H. Yun, Performance of ILU factorization preconditioners based on multisplittings, Numer. Math. 95 (2003), 761-779. 
YU DU HAN

Department of Mathematics

College of Natural Sciences

Chungbuk National University

Cheonguu 361-763, Korea

E-mail address: hanyd@hanmail.net

JAE HEON YUN

Department of Mathematics

College of Natural Sciences

Chungbuk National University

Cheonguu 361-763, Korea

E-mail address: gmjae@chungbuk.ac.kr 